GUIDELINES

\title{
Guidelines on the use of oesophageal dilatation in clinical practice
}

\author{
S A Riley, S E A Attwood
}

Gut 2004;53(Suppl I):i1-i6

\subsection{INTRODUCTION}

Oesophageal dilators or bougies have been used since the Middle Ages. Early bougies were made of natural materials and were used to disimpact food boluses by pushing them "blindly" into the stomach. The technique of oesophageal dilatation has evolved considerably in recent years. A range of purpose built dilators is now available, and with present day diagnostic techniques it is possible to select a dilator and dilatation technique appropriate to the clinical setting.

The relatively low morbidity and mortality of oesophageal dilatation has encouraged its widespread use. Despite this wealth of clinical experience however, the practice of oesophageal dilatation has been subject to surprisingly few controlled studies. The purpose of these guidelines is to highlight areas of good practice and promote the use of standardised protocols within and between centres.

\subsection{FORMULATION OF GUIDELINES}

These guidelines have been produced to conform to the North of England evidence based guidelines development project. ${ }^{1}{ }^{2}$ They are based on a Medline literature search using the search term "oesophageal dilatation" and on expert opinion and review. Although oesophageal dilatation may be performed during rigid oesophagoscopy and under radiological screening, this guidance relates primarily to oesophageal dilatation performed during flexible upper gastrointestinal endoscopy. Oesophageal dilatation in the paediatric population is considered outside the scope of these guidelines.

\subsection{Categories of evidence}

The strength of evidence used to formulate these guidelines was graded according to the following system:

- Ia-Evidence obtained from meta-analysis of randomised controlled trials.

- Ib-Evidence obtained from at least one randomised controlled trial.

- IIa-Evidence obtained from at least one well designed controlled study without randomisation.

- IIb-Evidence obtained from at least one other type of well designed quasi experimental study.

- III-Evidence obtained from well designed non-experimental descriptive studies such as comparative studies, correlation studies, and case studies.

- IV-Evidence obtained from expert committee reports or opinions, or clinical experiences of respected authorities.

The evidence category is indicated after the citations in the reference section at the end of these guidelines.

\subsection{Grading of recommendations}

The strength of each recommendation is dependent on the category of evidence supporting it, and is graded according to the following system:
- Grade A-requires at least one randomised controlled trial as part of the body of literature of overall good quality and consistency, addressing the specific recommendation (evidence categories Ia, Ib).

- Grade B-requires the availability of clinical studies without randomisation on the topic of recommendation (evidence categories IIa, IIb, III).

- Grade C-requires evidence from expert committee reports or opinions, or clinical experience of respected authorities, in the absence of directly applicable clinical studies of good quality (evidence category IV).

The grading category is indicated in the summary and recommendations section at the end of these guidelines.

\subsection{INDICATIONS}

Oesophageal dilatation is indicated in the treatment of symptomatic obstruction of the oesophagus. Obstruction may develop as a consequence of a wide range of anatomical and functional oesophageal disorders. Reflux induced strictures, malignant strictures, and achalasia are the most frequent indications but patients with anastomotic, sclerotherapy, radiation, medication, and corrosive induced strictures, and those with rings and webs frequently require dilatation. Patients with diffuse oesophageal spasm and other motility disorders may occasionally require dilatation of the lower oesophageal sphincter when conservative measures fail.

The primary aim of oesophageal dilatation is to alleviate symptoms, permit maintenance of oral nutrition, and reduce the risk of pulmonary aspiration. The technique may also be used to facilitate diagnostic gastroscopy when a stricture prevents passage of the endoscope, and to permit oesophageal stent insertion in the palliation of patients with oesophageal malignancy.

\subsection{CONTRAINDICATIONS}

Active oesophageal perforation is an absolute contraindication to oesophageal dilatation as it may extend the oesophageal defect and promote mediastinal soiling. The procedure should be undertaken with caution in those who have suffered a recent perforation or undergone recent upper gastrointestinal surgery.

The risks of dilatation are likely to be greater in patients with pharyngeal or cervical deformity and in those with large thoracic aneurysms. The risks of a perforation developing are greater during dilatation of malignant disease of the oesophagus.

Severe cardiorespiratory disease is a relative contraindication to all endoscopic procedures. The balance of risks and benefits should be individualised and carefully considered.

Major bleeding is uncommon following oesophageal dilatation but is more likely in patients with severe coagulopathy and in those taking anticoagulant drugs. 
Anticoagulants should be withdrawn and coagulopathy corrected prior to dilatation (see 5.5).

Concurrent radiotherapy is not a contraindication to oesophageal dilatation and mucosal biopsies do not prohibit dilatation during the same procedure. ${ }^{3}$

\subsection{PATIENT PREPARATION}

Oesophageal dilatation is best undertaken as a planned procedure in patients who have been appropriately investigated, prepared, and consented.

\subsection{Predilatation investigations}

The cause of oesophageal obstruction should be carefully assessed. Endoscopy and contrast radiology are both helpful and are often complimentary. Although some recommend a barium swallow examination in all patients presenting with dysphagia, many patients may be reasonably assessed by endoscopy alone. However, it should be remembered that patients with proximal dyshagia may be harbouring pathology which increases the risks of perforation-for example, pharygeal pouch, post-cricoid web-and in these circumstances endoscopy should only be undertaken by an experienced endoscopist.

Many oesophageal strictures will allow passage of a standard or narrow diameter gastroscope. The site and length of the stricture and the mucosal appearances should be documented. Features that may make dilatation more hazardous, such as angulation of the stricture and the presence of diverticulae, a hiatus hernia, or a small stomach, should also be noted. When a stricture prevents passage of the endoscope, a barium swallow examination will provide useful anatomical detail. This is particularly helpful in patients with long, tight, or complex strictures in whom dilatation may be technically difficult and associated with greater risks.

Biopsies or brush cytology should be taken for histological or cytological analysis. A tissue diagnosis is desirable, prior to dilatation, as it will influence overall management and the estimation of perforation risk. ${ }^{4}$ In patients with short, simple, benign looking strictures however, it is common practice for biopsies to be taken and dilatation performed during the same examination. When the stricture is tight or when the endoscopic features suggest malignancy, the results of biopsies are best awaited unless the patient has absolute dysphagia. Confirmation of malignancy should prompt a detailed assessment, including imaging to look for evidence of locoregional and distant spread. In patients with an inoperable tumour, dilatation may then be combined with stent insertion or other palliative procedures.

Patients with suspected achalasia should undergo detailed assessment to confirm the diagnosis and exclude occult carcinoma. The clinical features and endoscopic or barium swallow findings suggest the diagnosis but oesophageal manometry should be performed to confirm the diagnosis. Careful endoscopic assessment of the gastro-oesophageal junction should be performed to exclude malignancy. Computed tomography scanning and endosonography may be useful when a neoplasm is suspected and other modalities fail to confirm the diagnosis. ${ }^{5}$

\subsection{Information and consent}

Patients should receive information, giving details of oesophageal dilatation, prior to the procedure. It is particularly important that patients are aware of the perforation risk and that operative intervention may be required should perforation occur. Patients should also be informed of the therapeutic alternatives to oesophageal dilatation.

Written consent should be obtained in line with local hospital policies.

\subsection{Fasting}

All patients should be asked to fast for 4-6 hours to ensure an empty oesophagus and stomach during the procedure. Patients with achalasia are particularly prone to oesophageal stasis and may require a more prolonged fast or oesophageal lavage.

\subsection{Patient premedication}

Although some patients tolerate dilatation using only local anaesthesia, for many the procedure is uncomfortable and sedation is usually required. Patients with achalasia may find dilatation painful and the addition of an intravenous opiate is common practice.

The endoscopist is responsible for assessing the need for topical anaesthesia, sedation, and analgesia. The combination of topical anaesthesia and intravenous sedation may increase the risk of aspiration and some suggest the use of one or the other. ${ }^{6}$ Furthermore, the combination of intravenous sedation and analgesia is associated with adverse cardiorespiratory events and death during endoscopy. ${ }^{7}$ The elderly and those with coexistent cardiorespiratory, renal, and hepatic disease (ASA grades III-V) are at particular risk. Doses of sedation and analgesia should be kept to a minimum compatible with patient comfort and a successful procedure. Opioid and benzodiazepine antagonists should be immediately available.

\subsection{Patients taking oral anticoagulants and antiplatelet agents}

In patients taking oral anticoagulants, dilatation has the potential to produce bleeding, which may be difficult to control endoscopically. It is therefore advisable that patients at low risk of thromboembolic events should discontinue anticoagulants prior to the dilatation. A preprocedure prothrombin time should be performed. In patients at high risk of thromboembolic events, oral anticoagulants should again be discontinued prior to the procedure. Monitoring should be undertaken and intravenous heparin started once oral anticoagulation becomes subtherapeutic. Heparin should be discontinued 4-6 hours before dilatation and resumed 4-6 hours thereafter. Anticoagulants are generally resumed on the night of the procedure. ${ }^{8}$

The limited data available suggest that aspirin and nonsteroidal anti-inflammatory drugs do not increase the risk of significant bleeding after therapeutic endoscopy.

\subsection{Patients at risk of endocarditis}

Antibiotics should be given to patients with higher risk cardiac lesions (prosthetic heart valves, previous endocarditis, synthetic vascular grafts inserted within 12 months of the dilatation, and systemic pulmonary shunts) and those with neutropenia $\left(<100 \times 10^{9} / 1\right)$. The British Society of Gastroenterology guidelines on antibiotic prophylaxis during endoscopy should be followed. ${ }^{9}$

\subsection{THE DILATATION PROCEDURE}

\subsection{Personnel and experience}

Oesophageal dilatation is associated with clearly defined morbidity and mortality. It should only be performed by experienced endoscopists. Endoscopists who have performed less than 500 diagnostic procedures are four times more likely to cause perforation than their more experienced colleagues. ${ }^{4}$ All forms of therapeutic endoscopy should be taught only after adequate skills for diagnostic gastroscopy have been acquired (JAG recommend a minimum of 300 procedure ${ }^{10}$ ). Initially, oesophageal dilatation should be carried out only under direct supervision.

During oesophageal dilatation the endoscopist should be supported by at least two endoscopy assistants. Both have a 
role in monitoring patient comfort and safety throughout the procedure. They should be familiar with the endoscopic and dilatation equipment and should be capable of helping the endoscopist in case of an emergency, such as cardiorespiratory arrest.

Personnel trained in radiological techniques should be present when the procedure is performed under $x$ ray screening.

The principal risk of oesophageal dilatation is perforation. As patients who suffer perforation are best managed in conjunction with a surgeon, appropriate surgical support should be available.

\subsection{Oesophageal dilators}

Two types of oesophageal dilator are available: the push dilator (bougie) and the balloon dilator. ${ }^{11}$

\section{Push dilators}

Push dilators may be weighted (mercury or tungsten filled rubber bougies) or wire guided (metal olives, Celestin type dilators, or polyvinyl bougies).

Weighted bougies are available in a range of sizes (7$20 \mathrm{~mm}$ diameter). They may be passed blindly, under local anaesthetic, with the patient in a sitting position, and selected patients may be taught self dilatation.

Eder-Puestow dilators comprise a series of graduated metal olives (6.6-19.3 mm diameter) mounted on a flexible shaft. For many years it was the only system available for dilating resistant or complicated strictures. The system is durable and is said to be useful in patients with tortuous strictures or small stomachs.

Celestin dilators are long, tapered, radio-opaque bougies. There are two dilators, which increase in small steps, the larger to a maximum diameter of $18 \mathrm{~mm}$. They offer the advantage of full dilatation in two passages but because of their length should be used with care in patients with small stomachs and significant hiatus hernias.

Polyvinyl dilators have become popular in recent years. Savary Gillard dilators consist of a range of polyvinyl tubes (5-20 mm diameter), each with a $20 \mathrm{~cm}$ tapered tip. A radioopaque band at the widest point of the dilator aids radiological localisation. The American system is similar but dilators are impregnated with barium sulphate for easier radiological localisation and the distal tapering segment is shorter. Multiple passages may be required to achieve full dilatation.

\section{Balloon dilators}

Balloon dilators are widely used and may be passed through the scope or be wire guided. Balloon sizes range from 6 to $40 \mathrm{~mm}$, with the larger balloons reserved for the treatment of achalasia. The principal disadvantage of balloon dilators is their cost.

\subsection{The dilatation technique}

Prior to dilatation the endoscopist should consider five points:

(a) The diameter to which the obstruction should be dilated

In patients with benign peptic strictures, the results of dilatation appear best when a luminal diameter of $13-15 \mathrm{~mm}$ is achieved. This diameter is therefore usually recommended although greater diameters may be required when patients remain symptomatic. ${ }^{11}$ Large calibre dilators $(16-20 \mathrm{~mm}$ ) are advised in the treatment of patients with Schatzki's rings. ${ }^{12}$

In patients with achalasia the aim of oesophageal dilatation is to forcibly disrupt the lower oesophageal sphincter.
Dilators ranging from 30 to $40 \mathrm{~mm}$ are therefore usually employed.

In patients with malignant oesophageal disease however large calibre dilators are best avoided as perforation is more likely and dilatation is rarely the definitive treatment. Modest dilatation, sufficient to permit biopsy or endoscopic ultrasound or facilitate stent insertion, is the safest approach. In some patients expandable metal stents may be placed without the need for dilatation.

\section{(b) How quickly dilatation should be achieved}

Traditionally, it has been suggested that no more than three dilators of progressively increasing diameter $(3 \times 1 \mathrm{~mm}$ increments) should be passed in a single session to reduce the risk of perforation ("rule of three"). ${ }^{13}$ Several sessions were therefore often required to achieve adequate dilation. Recent experience suggests that the passage of a single large dilator ( $\geqslant 15 \mathrm{~mm}$ diameter) or incremental dilatation in larger steps may be safely employed in many patients with uncomplicated peptic strictures. ${ }^{14}$ However, it should be borne in mind that these observations were uncontrolled and it would be wise to employ a cautious approach in patients with tight, tough, or complex strictures.

\section{(c) The dilator that should be employed}

As balloon dilators generate only radial forces within a stricture, it has been suggested they are less likely to be associated with complications than the push dilators, which also generate longitudinal shearing forces. However, this has not been borne out by clinical studies and both push and balloon dilatations give good results in most situations. ${ }^{15-18}$

\section{(d) The need for wire guidance or endoscopic control} In most patients who require oesophageal dilatation it is desirable to use wire guided or endoscopically controlled techniques.

Most authorities agree that the unguided passage of weighted bougies should be restricted to the treatment of patients with simple reflux induced strictures, rings, or webs. Weighted bougies are not suited to the management of tight strictures. Dilators less than $10 \mathrm{~mm}$ in diameter are floppy and require radiographic screening to confirm passage through the stricture. ${ }^{19} 20$ Weighted bougies should not be used in the treatment of patients with complex strictures as perforations are more likely. ${ }^{21}$

Wire guided dilatation gives greater assurance that the dilator is following the line of the oesophageal lumen, thus reducing the risk of perforation. Routine radiological screening was previously recommended when undertaking wire guided dilatation but this is not essential when the anatomy is well defined, axial alignments are maintained, and the wire passes easily into the stomach. The guidewire should be placed at least $20-30 \mathrm{~cm}$ below the lowest point of the stricture, usually in the gastric antrum. Liberal lubrication facilitates passage of both the wire and dilator. Once positioned, the guidewire should be fixed externally to minimise the risk of internal displacement. Slight counter tension facilitates passage of the dilator. The dilator is withdrawn over the guidewire and the next dilator passed. Studies have shown that dilatation following endoscopic placement of a marked guidewire is a safe and effective technique, which negates the need for routine radiological screening. ${ }^{22} 23$

Through the scope balloon dilatation is performed under direct endoscopic visualisation. An adequate channel size and lubrication facilitate easy passage. The balloon should be centred at the tightest point of the stricture. Maximum dilating pressures vary in relation to balloon size and range between 30 and 45 psi but optimum pressure is unknown. 
Water is the standard agent used to expand the balloons although air or contrast agents may also be used. Recommended inflation times range from 20 to 60 seconds but again the optimum is unknown. A graded approach to dilatation is recommended by some with obliteration of the stricture waist seen during radiographic screening or endoscopic assessment of stricture dilatation as commonly used end points of success. Through the scope balloon dilatation has the additional advantage that it allows dilatation of the proximal part of a stricture. This may be helpful when a guidewire will not pass.

\section{(e) The need for radiographic screening}

The use of radiographic screening gives additional assurance and control of the dilatation process. During wire guided dilatation, it ensures that the wire has passed the stricture, that kinking of the wire has not occurred within or distal to the stricture and that, during the dilatation process, the dilator is following the line of the oesophageal lumen. During balloon dilatation, it indicates whether the balloon has slipped during inflation and whether obliteration of the stricture waist has occurred.

Radiographic screening is particularly helpful when the stricture is tortuous or complex or associated with a large hiatus hernia or diverticulae. It may also be of value when the guidewire meets with resistance during passage through the stricture or when an adequate length of wire cannot be passed distal to the stricture.

Although comparative trials are not available, the selective use of radiological screening appears safe and effective and is supported by extensive clinical experience. ${ }^{14}$

\subsection{Achalasia dilatation}

Several types of dilator are available for the treatment of achalasia. Comparative studies have shown similar results using different dilator systems and the choice should be based on training and experience. ${ }^{24} 25$

For achalasia, pneumatic dilators have become standard practice. ${ }^{26}{ }^{27}$ The Rigiflex system is widely used and comprises a cylindrical polyethylene balloon mounted on a thin flexible bougie passed over a guidewire. Radio-opaque rings mark the centre and ends of the balloon to facilitate placement using radiological screening. The dilators are available in three different balloon diameters $(3.0,3.5$, and $4.0 \mathrm{~cm})$ and a graded approach starting with the smallest dilator is recommended.

In practice, the balloon is first tested for leaks and asymmetry and a guidewire is then passed into the distal stomach through an endoscope. The endoscope is removed and the dilating assembly is passed over the guidewire. Radiological screening is traditionally employed but dilatation under direct endoscopic vision is also effective. ${ }^{28}$

The optimal distension pressure is not known (pressures between 7 and 20 psi are frequently employed). High and low compliance balloons appear to be equally effective. ${ }^{29}$ Obliteration of the waist imposed by the lower oesophageal sphincter on the balloon (seen during radiological screening during slow inflation of the balloon) is thought critical by many. At the initial dilatation, the $3.0 \mathrm{~cm}$ balloon, inflated for short periods (6-15 seconds), appears as effective as larger balloons inflated for longer durations. ${ }^{30-32}$

\subsection{Monitoring during the procedure}

The patient's clinical condition should be observed throughout the procedure by both the endoscopist and nursing staff. Supplemental oxygen and pulse oximetry should be used routinely as dilatation is frequently performed in high risk patients, is occasionally prolonged, and some patients require both opioids and benzodiazepines. Monitoring should be continued into the recovery period.

\subsection{AFTERCARE}

Patients should be closely observed after oesophageal dilatation and pulse, blood pressure, and temperature should be measured regularly. Oesophageal dilatation is now frequently undertaken as an outpatient procedure, and this appears safe providing the procedure is routine and the patient is closely observed after the procedure..$^{34}$ It is important to allow sufficient time for recovery, for the patient to have a drink (initially water), and to be assessed by appropriately trained staff (routine observations and a check for surgical emphysema). Patients with dysphagia are therefore best examined at the beginning of the endoscopy list. Facilities should be available to keep a patient overnight for observation.

Following oesophageal dilatation, particularly for achalasia, some recommend a chest $x$ ray and contrast study to exclude perforation. These investigations are not essential but should be performed urgently in patients who develop pain, breathlessness, fever, or tachycardia. As perforation risks are higher following dilatation for achalasia, many recommend a period of overnight observation.

On leaving hospital patients should be well and tolerating oral fluids. All patients should receive written information indicating the need to return immediately should they develop pain or breathlessness or become unwell.

\subsection{COMPLICATIONS}

The principal complications of oesophageal dilatation are perforation, pulmonary aspiration, and bleeding. ${ }^{35}$

A UK regional audit reported an overall perforation rate of $2.6 \%$ with a mortality of $1 \% .{ }^{4}$ Perforation was less common following dilatation of benign strictures $(1.1 \%$ with a mortality of $0.5 \%$ ) than following dilatation and/or intubation of malignant strictures $(6.4 \%$ with a mortality of $2.3 \%)$. Elderly patients appeared more at risk. The risks are also greater when the endoscopist is inexperienced and when strictures are complex, particularly when weighted bougies are passed blindly. ${ }^{21}$

Perforation usually occurs at the site of the stricture resulting in intra-abdominal or intra-thoracic perforation, the latter being more serious. Although perforation is often linked to the use of large dilators it may complicate the passage of a small dilator or be caused by the guidewire.

Perforation should be suspected when patients develop pain, breathlessness, fever, or tachycardia. Transient chest pain is not uncommon following dilatation but persistent pain should prompt a chest $x$ ray and contrast study to look for perforation. Some recommend endoscopic reinspection immediately on completion of the dilatation procedure as the appearances may raise the possibility of perforation and prompt early treatment. A chest $x$ ray may show pneumomediastinum, pneumothorax, air under the diaphragm, or a pleural effusion but normal appearances do not exclude perforation and, if there is any clinical suspicion, a water soluble contrast study should be performed. Iatrogenic perforation is a medical emergency. The patient should be assessed by an experienced physician and experienced surgeon in order to formulate an appropriate management plan.

The risk of perforation in achalasia is reported as $0-7 \%$ (mostly $3-4 \%$ ) with a mortality of $<1 \%$. The perforation rate may be lower with a graded approach to balloon dilatation but most perforations occur during the first dilatation. ${ }^{36-38}$ Post-dilatation reflux may occur but is usually mild and readily controlled with acid suppression. 


\subsection{OUTCOMES AND FOLLOW UP}

Most patients respond well to oesophageal dilatation but outcomes are influenced by the underlying pathology. In patients with benign reflux induced strictures, a graded stepwise approach to between 13 and $20 \mathrm{~mm}$ gives good relief in $85-93 \%$ of cases. Dilatation appears less effective in those with radiation or corrosive induced strictures.

Some patients require repeat dilatation after an initial successful dilatation. ${ }^{39-41}$ Predictors for repeated dilatation are "non-peptic" causes of stricture, fibrous strictures, and a maximum dilator size less than $14 \mathrm{~mm}$. Tight strictures may require short interval redilatation to ensure a reasonable duration of response. Weekly dilatation until easy passage of a greater than $14 \mathrm{~mm}$ dilator is a common strategy. Carefully selected patients with recurrent benign strictures may be taught self dilatation. ${ }^{42}$

The need for redilatation in patients with peptic strictures is much reduced when maintenance acid suppression is prescribed following the initial dilatation. ${ }^{43}{ }^{44}$ Standard dose proton pump inhibitor treatment is clearly more effective than $\mathrm{H}_{2}$ receptor antagonists. Twice daily dosing with proton pump inhibitors may be required when restenosis occurs rapidly. Further biopsies and imaging are also recommended under these circumstances to exclude occult malignancy.

Although oesophageal dilatation is effective in most patients with benign strictures, a small number require surgical intervention. Patients who need frequent dilatation despite proton pump inhibitor treatment and those who are technically difficult to dilate should be considered for operative treatment by antireflux surgery. ${ }^{45}$

Most malignant strictures respond to dilatation but relief is usually short lived and more definitive treatment is necessary. Patients with malignant strictures should undergo imaging to assess the degree of locoregional and distant spread. Although there is as yet no clear consensus about the best palliation of malignant strictures, expandable metal stents have become popular and dilatation is often combined with stent insertion. The dysphagia caused by extrinsic compression of the oesophagus responds poorly to oesophageal dilatation.

Recent studies report that $58-95 \%$ of patients with achalasia have excellent to good results following pneumatic dilatation. If a single session does not produce satisfactory results, a second and third attempt may be appropriate before considering the surgical option. Younger patients may respond less well to balloon dilatation. ${ }^{46}{ }^{47}$

Patients with achalasia should be aware of the other therapeutic options. Surgical cardiomyotomy generally provides high rates of symptomatic relief, although this has to be balanced against operative risks and the problem of long term reflux. ${ }^{48}$ The surgical approach may be by open or laparoscopic routes. Botulinum toxin may be considered in elderly patients and those at high surgical risk but long term results are modest and repeated injection often required. ${ }^{49}$

\subsection{AUDIT}

Many units perform relatively small numbers of oesophageal dilatations. In order to concentrate expertise, consideration should be given to limiting the number of clinicians who perform oesophageal dilatation. Audit standards should focus on the adequacy of predilatation investigation, consent procedures, and complication rates, notably perforation rates and mortality.

\subsection{Summary and recommendations}

(1) Oesophageal dilatation is indicated in the treatment of symptomatic oesophageal obstruction. When performed appropriately and carefully it is a highly effective technique with a low morbidity and mortality (grade B).

(2) Oesophageal dilatation should be undertaken as a planned procedure where possible in patients who have been adequately investigated and prepared. Endoscopy and contrast radiology are helpful and often complimentary. A tissue diagnosis is desirable prior to dilatation. Patients with suspected achalasia should be investigated to confirm the diagnosis and exclude occult malignancy (grade C).

(3) Informed consent should be obtained prior to upper gastrointestinal endoscopy and if dilatation is planned the risks and outcomes discussed. The patient should understand the perforation risk and the possible need for surgical intervention should perforation occur. Patients should be aware of the alternatives to dilatation (grade C).

(4) Local anaesthesia or intravenous sedation with or without intravenous analgesia should be given in accordance with the British Society of Gastroenterology guidelines on sedation during endoscopy. At risk patients should be identified before the procedure and monitored carefully throughout (grade C).

(5) Oral anticoagulants should be discontinued prior to oesophageal dilatation. In those at high risk of thromboembolism, heparin should be started when oral anticoagulation becomes subtherapeutic. Heparin should be discontinued 4-6 hours prior to the procedure and restarted 4-6 hours afterwards (grade C).

(6) Antibiotics should be given to patients with higher risk cardiac lesions and neutropenia in line with British Society of Gastroenterology guidelines (grade C).

(7) Oesophageal dilatation should only be undertaken by experienced endoscopists (grade B). The endoscopist should be supported by two assistants during the procedure. Surgical expertise should be available in case of perforation. (8) Both push dilators (bougies) and balloon dilators give good results (grade A). Results appear best when a luminal diameter of 13-15 mm is achieved. The passage of a single large dilator appears safe in simple uncomplicated strictures but a cautious graded approach is recommended in patients with tight, tough, or complex strictures (grade B). In most cases it is desirable to use either wire guided or endoscopically controlled techniques. The addition of radiographic screening is helpful when the stricture is tortuous or complex or associated with large hiatus hernia or diverticulae and when difficulty is encountered passing the guidewire (grade C).

(9) Most patients with achalasia respond well to pneumatic dilatation of the lower oesophageal sphincter. Optimal distension pressures are unknown but the $3.0 \mathrm{~cm}$ balloon inflated for a short period appears effective (grade A).

(10) Patients should be closely observed following oesophageal dilatation. In uncomplicated cases the procedure may be safely performed as an outpatient (grade B). Perforation should be suspected and a chest $x$ ray and contrast study should be performed urgently in patients who develop pain, breathlessness, fever, or tachycardia.

(11) Oesophageal dilatation is often only one part of the management of the patient. Those with malignant strictures for example, require detailed assessment by a multidisciplinary team (grade C), those with reflux induced strictures require maintenance acid suppression therapy with standard or high dose proton pump inhibitors to reduce the need for further dilatation (grade A).

(12) Audit standards should focus on the adequacy of investigations, consent procedures, and complication rates, notably perforation and mortality. 


\section{Authors' affiliations}

S A Riley, Sheffield Teaching Hospitals, Northern General Hospital, Sheffield S5 7AU, UK

S E A Attwood, Hope Hospital, Stott Lane, Salford, Manchester M6 8HD, UK

Correspondence to: Dr S A Riley, Department of Gastroenterology Sheffield Teaching Hospitals, Northern General Hospital, Sheffield'S5 7AU, UK; stuart.riley@sth.nhs.uk

\subsection{REFERENCES}

1 Eccles M, Clapp Z, Grimshaw J, et al. North of England evidence based guidelines development project: methods of guidelines development. $\mathrm{Br}$ Med J 1996;312:760-2, (IV)

2 Grimshaw J, Eccles M, Russell I. Developing clinically valid practice guidelines. J Eval Clin Pract 1995;1:37-48, (IV).

3 BarkinJ S, Taub S, Rogers Al. The safety of combined endoscopy, biopsy and dilatation in oesophageal strictures. Am J Gastroenterol 1981;76:23-6, (III).

4 Quine MA, Bell GD, McCloy RF, et al. Prospective audit of perforation rates following upper gastrointestinal endoscopy in two regions of England. Br J Surg 1995;82:530-3, (III)

5 Vaezi MF. Achalasia: diagnosis and management. Semin Gastrointest Dis 1999;10:103-12, (III).

6 Quine MA, Bell GD, McCloy RF, et al. Prospective audit of upper GI endoscopy in two regions of England; safety, staffing and sedation methods. Gut 1995; 36:462-7, (III)

7 Bell GD, McCloy RF, Charlton JE, et al. Recommendations for standards of sedation and patient monitoring during gastrointestinal endoscopy. Gut 1991;32:823-7, (IV).

8 Guideline on the management of anticoagulation and antiplatelet therapy for endoscopic procedures, ASGE publication 1029, December, 1997 (IV).

9 Antibiotic prophylaxis in gastrointestinal endoscopy. London: British Society of Gastroenterology, January, 2001 (www.bsg.org.uk).

10 Guidelines for the training, appraisal and assessment of trainees in GI endoscopy, Joint Advisory Group on Gastrointestinal Endoscopy, 2001 (www.thejag.org.uk).

11 Bennet JR, Cox JG. Benign oesophageal strictures. In: Bennet JR, Hunt RH, eds. Therapeutic endoscopy and radiology of the gut, 2nd edn. London: Chapman and Hall Medical, 1990:11.

12 Eckhardt VF, Kanzler G, Willems D. Single dilatation of symptomatic Schatzki's rings. A prospective evaluation of its effectiveness. Dig Dis $S_{c i}$ 1992:37:577-82.

13 Langdon DF. The rule of three in oesophageal dilatation. Gastrointest Endosc 1997;45:111.

14 Kozarek RA, Patterson DJ, Ball TJ, et al. Esophageal dilation can be done safely using selective fluoroscopy and single dilating sessions. J Clin Gastroenterol 1995:20:184-8, (IV).

15 Cox JG, Winter RK, Maslin SC, et al. Balloon or bougie for dilatation of benign esophageal stricture? Dig Dis Sci 1994;3:776-81, (Ib).

16 Saeed ZA, Winchester CB, Ferro PS, et al. Prospective randomized comparison of polyvinyl bougies and through-the-scope balloons for dilation of peptic strictures of the esophagus. Gastrointest Endosc 1995;41:189-95, (Ib).

17 Scolapio JS, Pasha TM, Gostout CJ, et al. A randomized prospective study comparing rigid to balloon dilators for benign esophageal strictures and rings. Gastrointest Endosc 1999:50:13-17, (lb).

18 Yamamoto H, Hughes RW, Schroeder KW, et al. Treatment of benign oesophageal stricture by Eder-Puestow or balloon dilators; a comparison between randomised and prospective non-randomised trials. Mayo Clin Proc 1992:67:228-36, (la).

19 McClare SA, Wright RA, Brady PG. Prospective randomised study of Maloney oesophageal dilation-blinded versus fluoroscopic guidance. Gastrointest Endosc 1990;36:272-5, (lb).

20 McClare SA, Brady PG, Wright RA, et al. Does fluoroscopic guidance for Maloney oesophageal dilation impact on the clinical endpoint of therapy relief of dysphagia and achievement of luminal patency? Gastrointest Endosc 1996;43:93-7, (Ib)

21 Hernandez $\mathrm{L}$, Jacobson JW, Harris MS. Comparison among the perforation rates of Maloney, balloon and savary dilation of esophageal strictures. Gastrointest Endosc 2000;51:460-2, (III).
22 Fleischer DE, Benjamin SB, Cattau EL, et al. A marked guidewire facilitates oesophageal dilation. Am J Gastroenterol 1989;84:359-61, (III).

23 Kadakia SC, Parker A, Carrougher JG, et al. Oesophageal dilation with polyvinyl bougies using a marked guidewire without the aid of fluoroscopy: an update. Am J Gastroenterol 1993:88:1381-6, (III).

24 Stark GA, Castell DO, Richter JE, et al. Prospective randomised comparison of Brown-McHardy and Microvasive balloon dilators in treatment of achalasia. Am J Gastroenterol 1990;85:1322-6, (llb).

25 Mearin F, Armengol JR, Chicharro L, et al. Forceful dilation under endoscopic control in the treatment of achalasia: a randomised trial of pneumatic versus metallic dilator. Gut 1994;35:1360-2, (III).

26 Katz PO, Gilbert J, Castell DO. Pneumatic dilatation is effective long-term treatment for achalasia. Dig Dis Sci 1998;43:1973-7, (IV).

27 Fellows IW Ogilvie AL, Atkinson M. Pneumatic dilatation in achalasia. Gut 1983;24:1020-3, (III)

28 Lambroza A, Schuman RW. Pneumatic dilation for achalasia without fluoroscopic guidance: safety and efficacy. Am J Gastroenterol 1995:90:1226-9, (IV).

29 Muehldorfer SM, Hahn EG, Ell C. High and low compliance balloon dilators in patients with achalasia: a randomised prospective comparitive trial. Gastrointest Endosc 1996;44:398-403, (b).

30 Gideon RM, Castell DO, Yarze J. Prospective randomized comparison of pneumatic dilatation technique in patients with idiopathic achalasia. Dig Dis Sci 1999;44:1853-7, (Ib)

31 Khan AA, Shah SW, Alam A, et al. Pneumatic balloon dilation in achalasia: a prospective comparison of balloon distension time. Am J Gastroenterol 1998;93:1064-7, (lb).

32 Gideon RM, Katzka D, Yarze J, et al. Prospective comparison of inflation technique for pneumatic dilation of achalasia. Am J Gastroenterol 1995;90:1559, (IV)

33 Barkin JS, Guelrud M, Reiner DK, et al. Forceful balloon dilation: an outpatient procedure for achalasia. Gastrointest Endosc 1990:36:123-6, (IV).

34 Bradpiece HA, Galland RB, Murray J, et al. Oesophageal dilation as an outpatient procedure. Surg Gynaecol Obstet 1988;167:45-8, (III).

35 Tulman AB, Boyce HW. Complications of oesophageal dilation and guidelines for their prevention. Gastrointest Endosc 1981; 27:229-34, (III).

36 Metman EH, Lagasse JP, d'Alteroche L, et al. Risk factors for immediate complications after progressive pneumatic dilation for achalasia. Am J Gastroenterol 1999;94:1 179-85, (III).

37 Nair LA, Reynolds JC, Parkman HP, et al. Complications during pneumatic dilation for achalasia or diffuse oesophageal spasm. Analysis of risk factors, early clinical characteristics and outcome. Dig Dis Sci 1993;38:1893-904, (III).

38 Borotto E, Gaudric M, Danel B, et al. Risk factors of oesophageal perforation during pneumatic dilatation for achalasia. Gut 1996;39:9-12, (III).

39 Ogilvie AL, Ferguson R, Atkinson M. Outlook with conservative treatment of peptic oesophageal stricture. Gut 1980;21:23-5, (III).

40 Wesdorp IC, Bartelsman JF, den Hartog Jager FC, et al. Results of conservative treatment of benign oesophageal strictures: a follow-up study in 100 patients. Gastroenterology 1982;82:487-93, (III).

41 Patterson DJ, Graham DY, Smith JL, et al. Natural history of benign oesophageal stricture treated by oesophageal dilation. Gastroenterology 1983;85:346-50, (III)

42 Grobe JL, Kozarek RA, Sanowski RA. Self-bougienage in the treatment of benign oesophageal stricture. J Clin Gastroenterol 1984;6:109-12, (III).

43 Smith PM, Kerr GD, Cockel R, et al. A comparison of omeprazole and ranitidine in the prevention of recurrence of benign esophageal stricture. Restore Investigator Group. Gastroenterology 1994;107:1312-18, (1b).

44 Swarbrick ET, Gough AL, Foster CS, et al. Prevention of recurrence of oesophageal stricture, a comparison of lansoprazole and high-dose ranitidine. Eur J Gastroenterol Hepat 1996;8:413-8, (Ib).

45 Watson A. Reflux stricture of the oesophagus. Br J Surg 1987;74:443-8, (IV).

46 Kadakia SC, Wong RK. Graded pneumatic dilation using Rigiflex achalasia dilators in patients with primary oesophageal achalasia. Am J Gastroenterol 1993;88:34-8, (III).

47 Eckardt VF, Aignherr C, Bernhard G. Predictors of outcome in patients with achalasia treated by pneumatic dilation. Gastroenterology 1992;103:1732-8, (III).

48 Csendes A, Braghetto I, Henriquez A, et al. Late results of a prospective randomised study comparing forceful dilation and oesophagomyotomy in patients with achalasia. Gut 1989;30:299-304, (lb)

49 Vaezi MF, Richter JE, Wilcox CM, et al. Botulinum toxin versus pneumatic dilatation in the treatment of achalasia: a randomised trial. Gut 1999;44:231-9, (lb) 